\title{
The Periodontal Pathogen Aggregatibacter Actinomycetemcomitans Deteriorates Ventricular Remodeling After Myocardial Infarction in Mice
}

\author{
Tomoya Hanatani,,${ }^{1}$ DDS, Jun-ichi Suzuki,${ }^{2}$ MD, Masahito Ogawa,${ }^{2}$ MS, Norio Aoyama, ${ }^{1}$ DDS, \\ Naho Kobayashi, ${ }^{1}$ DDS, Yasunobu HiRata, ${ }^{2}$ MD, Ryozo NaGaI,${ }^{3}$ MD, Yuichi Izumi, ${ }^{1}$ DDS, \\ and Mitsuaki IsoBE, ${ }^{4} \mathrm{MD}$
}

\begin{abstract}
SUMMARY
Chronic inflammation plays a fundamental role in coronary heart disease (CHD). Periodontal disease is a common infectious disease and is a potential source of systemic inflammation. However, the effect of periodontal infection on $\mathrm{CHD}$ has not yet been proven. The purpose of this study was to determine the effect of periodontopathic bacteria on experimental myocardial infarction (MI). We implanted a chamber into the subcutaneous tissue of each male mouse. Aggregatibacter actinomycetemcomitans (A.a. $n=8)$, which is a major periodontal pathogen, or PBS $(n=6)$ was injected into the chamber. Then, MI was induced by permanent ligation of the left anterior descending coronary artery. To exclude the nonspecific effect of the pathogen, we injected A.a. into the mice without MI $(n=4)$. The plasma level of antiA.a. antibody was statistically higher in A.a.-infected mice than in vehicle control mice. Seven days after the myocardial ischemia, the A.a.-positive MI hearts showed a larger infarct size and length than the A.a.-negative MI mice. The A.a.positive MI hearts showed more MOMA-2 positive myocardial infiltrating cells compared to the A.a.-negative MI mice. The injection of A.a. into the mice without MI did not affect their hearts. We concluded that a periodontal pathogen infection might deteriorate ventricular remodeling after MI through inflammatory cell infiltration. (Int Heart J 2012; 53:
\end{abstract} 253-256)

Key words: Periodontitis, Bacterial infection, Myocardial infarction, Inflammation, Ventricular remodeling

$\mathrm{M}$ yocardial infarction (MI) leads to complex structura alterations of the ventricular myocardium, which is known as pathological remodeling. ${ }^{1-4)}$ MI and the subsequent ventricular remodeling is the most common cause of chronic heart failure. ${ }^{5)}$ It is hypothesized that prolonged activation of proinflammatory factors leads to myocardial damage and dysfunction. ${ }^{6-8)}$ Although chronic inflammatory factors are key mediators of post-MI cardiac repair and adverse remodeling, ${ }^{9)}$ the details of the mechanisms still need to be elucidated.

Periodontitis is known to be a risk factor for development of cardiovascular diseases. ${ }^{10)}$ More than 700 different bacterial species have been detected in samples from subgingival plaque biofilm and other sites in the oral cavity. ${ }^{11)}$ Periodontitis is a chronic inflammatory condition associated with infection with predominantly Gram-negative bacteria. Bioactive molecules released from pathogenic microorganisms located in the subgingival biofilm enhance inflammatory responses. ${ }^{12)}$ The global prevalence of periodontal disease is high, and severe forms of chronic periodontitis affect about $15 \%$ of individuals world- wide. ${ }^{13)}$ Aggregatibacter actinomycetemcomitans (A.a.) is a Gram-negative bacterium present in the oral cavity of one-third or more of the population. A.a. has also been isolated from numerous other diseased sites of the body and is considered a systemic pathogen. ${ }^{14,15)}$ We previously reported that A.a. was found in oral samples in patients with acute coronary syndrome (ACS), whereas no A.a. was found in chronic coronary heart disease (CHD) patients. We also reported higher serum titers of immunoglobulin G against A.a. in ACS patients compared to patients with chronic CHD. ${ }^{16)}$ However, no report has examined the pathological effect of A.a. on ventricular remodeling after MI. Therefore, to clarify the relationship between A.a. and MI, we used a murine MI model with systemic A.a. infection.

\section{Methods}

Animal model: We used a subcutaneous chamber model as

From the ${ }^{1}$ Department of Periodontology, Graduate School of Medical and Dental Sciences, Tokyo Medical and Dental University, Departments of ${ }^{2}$ Advanced Clinical Science and Therapeutics and ${ }^{3}$ Cardiovascular Medicine, The University of Tokyo, and ${ }^{4}$ Department of Cardiovascular Medicine, Tokyo Medical and Dental University, Tokyo, Japan.

This study was supported by grants from the Takeda Science Foundation, Medical Survey and Research Foundation, Mitsui Life Insurance Research Foundation, Mitsui Sumitomo Marine Welfare Research Foundation, Geriatric Dental Research Foundation, Human Health Future Research Foundation, St. Luke's Hospital Research Foundation, and the Japan Society for the Promotion of Science (JSPS) through its "Funding Program for World-Leading Innovative R\&D on Science and Technology (FIRST Program)".

Address for correspondence: Jun-ichi Suzuki, MD, Department of Advanced Clinical Science and Therapeutics, The University of Tokyo, 7-3-1 Hongo, Bunkyo-ku, Tokyo 113-8655, Japan.

Received for publication January 10, 2012

Revised and accepted April 23, 2012. 
previously described. ${ }^{17)}$ Male C57BL/6J mice (7 weeks old) were used in these studies. Coil-shaped chambers were prepared from 0.5 -mm stainless-steel wire and surgically implanted into the dorsolumbar region subcutaneously. After the healing period, the chambers were used as a biological compartment for inducing inflammation. This investigation conformed to the Guide for the Care and Use of Laboratory Animals of the Tokyo Medical and Dental University.

Bacterial growth and immunization: A.a., strain Y4, was revived by culturing on TSBV agar plates and incubated in 5\% $\mathrm{CO}_{2}$ at $37^{\circ} \mathrm{C}$ for 3 to 5 days. The purity of the cultures was checked under a phase-contrast microscope. Bacterial cells were placed into peptone yeast extract for 1 to 2 days. The bacterial concentrations were standardized to $10^{8}$ colony-forming units (CFUs)/mL. Mice were injected with live A.a. (0.1 $\mathrm{mL}) 2$ weeks after chamber implantation and subjected to surgery one week later. The level of anti-A.a. specific IgG in the plasma was determined by an enzyme-linked immunosorbent assay (ELISA). The plasma samples were obtained before coil implantation and sacrifice of the mice.

Myocardial infarction: MI was produced as previously described. Briefly, mice were anesthetized with $3.6 \%$ chloral hydrate $(10 \mathrm{mg} / 100 \mathrm{~g}$ body weight) and intubated with a small rodent respirator (MiniVent 845, Harvard Apparatus). The chest was opened by a left lateral thoracotomy and the left anterior descending (LAD) coronary artery was ligated using 8/0 nylon suture after removal of the pericardium. We defined phosphate buffered saline (PBS) injected MI mice as A.a.-negative MI mice and A.a. injected MI mice as A.a.-positive MI mice. To exclude the nonspecific effect of the pathogen, we injected A.a. into the sham operated mice without MI $(n=4)$. Mice were studied for up to 7 days after the MI and then sacrificed. Native mice were used for the control study.

Hemodynamic measurements and echocardiogram: Arterial blood pressure (BP) and heart rate (HR) were measured using a tail-cuff system (BP-98A, Softron Co., Tokyo). Transthoracic echocardiography was performed on animals anesthetized by intraperitoneal administration of $3.6 \%$ chloral hydrate (10 $\mathrm{mg} / 100 \mathrm{~g}$ body weight). An echocardiograph with a $14-\mathrm{MHz}$ transducer (Toshiba, Tokyo) was used for recording. A 2D targeted M-mode and B-mode echocardiogram was obtained along the short-axis view of the left ventricle at the papillary muscles. Left ventricular end-diastolic and end-systolic dimension (LVDd and LVDs) and fractional shortening (FS) were calculated from M-mode echocardiograms over three consecutive cardiac cycles according to the American Society for Echocardiography leading edge method. We averaged the measurements of 3 consecutive cardiac cycles. Measurements were made offline by two independent investigators.

Histopathology: Hearts were harvested immediately after the mice were killed on day 7 after MI. Heart weight and body weight were measured to calculate the heart to body ratio. Three transverse sections per heart were obtained for histological examination. Apex, mid, and basal level slices were stained with Mallory stain. Blue staining of collagen fibers was quantified as a measure of fibrosis using Image-Pro Express software. The ratios of infarction area per whole myocardium area and the ratios of infarction length per whole left ventricular inner circumference were calculated.

Immunohistochemistry: The sections were incubated with primary antibodies against MOMA-2 (BMA Biomedicals AG,
Switzerland) or monocyte chemoattractant protein (MCP)-1 (Santa Cruz Biotechnology, Santa Cruz, USA) at $4^{\circ} \mathrm{C}$ for 12 hours. Antibody-HRP conjugate was detected with a Histofine Simplestain Kit (Nichirei Corporation, Tokyo) according to the manufacturer's protocol. Enzyme activity was detected with 3-amino-9-ethylcarbazole. The positive cell numbers in each sample were counted and the numbers averaged. ${ }^{18)}$

Statistical analysis: Groups were compared using the Student $t$ test. A value of $P<0.05$ was considered to be statistically significant.

\section{RESULTS}

Plasma anti-A.a. antibody levels: The A.a.-positive MI mice showed significantly higher plasma anti-A.a. antibody levels than the A.a.-negative MI mice (Figure 1).

Organ weights: The heart weight to body weight ratio was comparable between the native mice and the A.a.-negative MI mice. The ratio was also comparable between the A.a.-negative MI mice and the A.a.-positive MI mice (data not shown).

Heart rate and blood pressure: $\mathrm{HR}$ and mean $\mathrm{BP}$ were not significantly different among the native mice, the A.a.-negative MI mice, and the A.a.-positive MI mice during the observation period (data not shown).

Echocardiogram: LVDd and LVDs increased in the A.a.-negative MI mice compared to the native mice. It is noteworthy that both diameters (LVDd and LVDs) showed an additional increase in the A.a.-positive MI mice compared to the A.a.negative MI mice (Figure 2). FS was comparable between the A.a.-positive MI mice and the A.a.-negative MI mice.

Histopathology: The A.a.-negative MI hearts showed left ventricular fibrosis that was induced by myocardial infarction. Of note, the A.a.-positive MI hearts showed larger ratios of infarction area per whole myocardium area and the ratios of infarction length per whole left ventricular inner circumference than A.a.-negative MI mice (Figure 3). A.a. injection into the mice without MI showed no myocardial ischemic and fibrotic changes. This was a comparable result to the results for hearts from native control mice (Figure 4).

Immunohistochemistry: The A.a.-negative MI hearts showed moderate MOMA-2 positive infiltrating cells at the border area

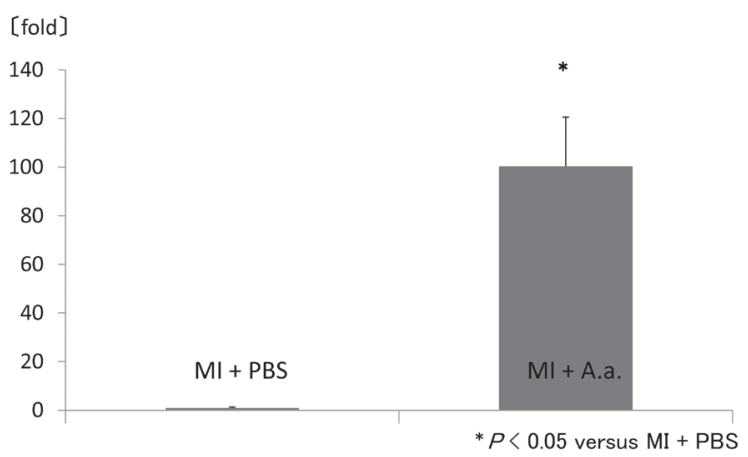

Figure 1. Plasma anti-A.a. antibody levels. Plasma anti-A.a. antibody levels are shown. The A.a.-positive MI mice showed significantly higher plasma anti-A.a. antibody levels than the A.a.-negative MI mice. Values are expressed as the mean $\pm \mathrm{SE} .{ }^{*} P<0.05$ versus A.a.-negative MI mice. 

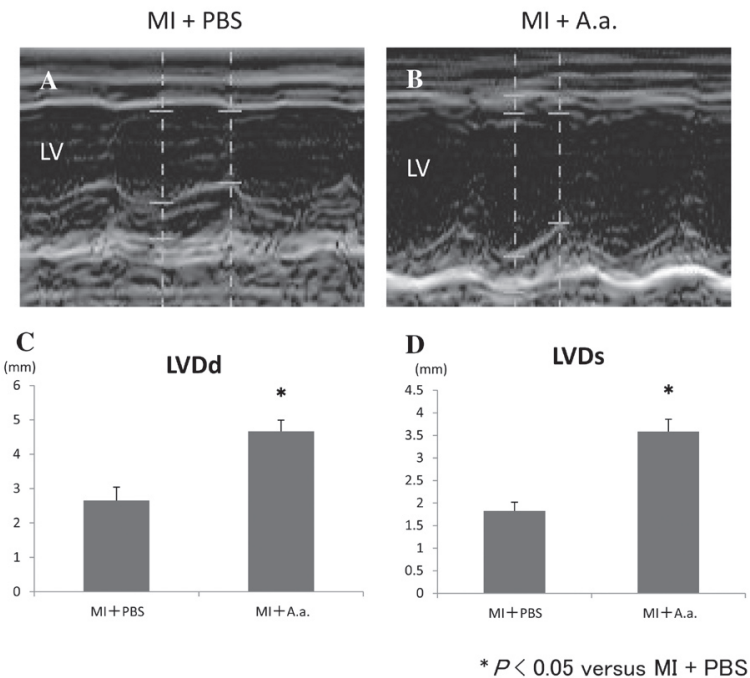

Figure 2. Echocardiogram. Representative M-mode echocardiogram (A and $\mathbf{B})$ and quantitative data (C and $\mathbf{D})$ are shown. LVDd and LVDs increased in the A.a.-positive MI mice compared to the A.a.-negative MI mice. Values are expressed as the mean \pm SE. ${ }^{*} P<0.05$ versus A.a.-negative MI mice.
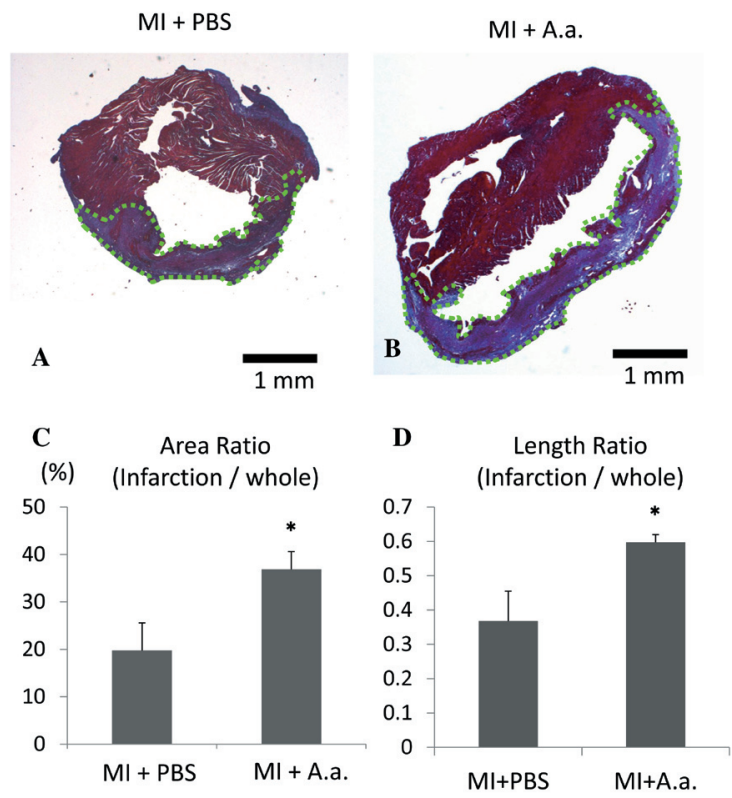

Figure 3. Histopathology. Representative Mallory staining (A and B) and quantitative results of the ratios of infarction area per whole myocardium area $(\mathbf{C})$ and the ratios of infarction length per whole left ventricular inner circumference (D) are shown. Green dots show fibrotic areas. Values are expressed as the mean \pm SE. ${ }^{*} P<0.05$ versus A.a.-negative MI mice.

of the myocardial ischemia, while native mouse hearts showed no MOMA-2 positive cells. It is noteworthy that the A.a.-positive MI hearts had large numbers of the MOMA-2 positive cells at the border area of the ischemic myocardium compared to the A.a.-negative MI hearts. They were observed not only at the border area of the ischemia but also at myocardial areas re-
A

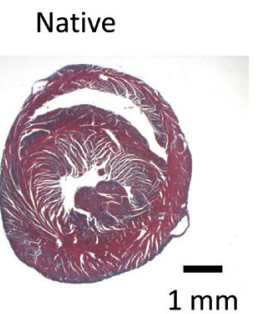

B

Non-MI+ A.a.

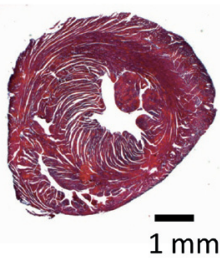

Mallory staining, $\times 20$

Figure 4. Representative heart samples with Mallory staining are shown. A.a. injection into the mice without MI (B) showed no myocardial ischemic or fibrotic changes. This was a comparable result to that for hearts from native control mice (A).
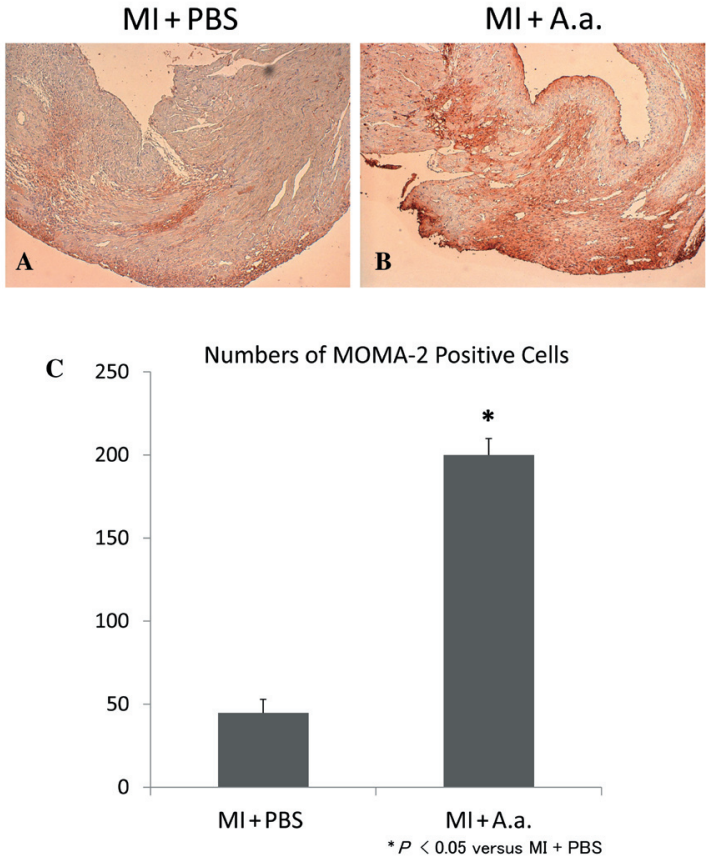

Figure 5. Immunohistochemistry detecting MOMA-2 positive cells. Representative immunohistochemical MOMA-2 staining of A.a.-negative MI mouse heart (A), A.a.-positive MI mouse heart (B), and quantitative data (C) are shown. Values are expressed as the mean \pm SE. ${ }^{*} P<0.05$ versus A.a.-negative MI mice.

mote from the ischemia. Statistically, the A.a.-positive MI mice showed a greatly increased number of MOMA-2 positive cells compared to the A.a.-negative MI hearts (Figure 5). In contrast, the A.a.-positive MI hearts showed comparable MCP-1 positive cells to the A.a.-negative MI hearts.

\section{Discussion}

Pathological myocardial ventricular remodeling is a chronic process which influences cardiac function and patient 
outcome after MI. ${ }^{19)}$ Although a strong association between dental disease and MI has been demonstrated, ${ }^{20)}$ the pathological mechanism remains unknown. Recently, we revealed that A.a. was frequently detected in patients with ACS. ${ }^{16)}$ In this study, we, for the first time, have demonstrated that A.a. infection accelerated the progression of ventricular remodeling after MI using a mouse model. Our results imply that periodontitis deteriorates MI-induced myocardial ventricular remodeling.

It is well known that bacterial components affect endothelial integrity, ${ }^{21,22)}$ the metabolism of plasma lipoprotein, ${ }^{23)}$ blood coagulation, ${ }^{24,25)}$ and platelet function and prostaglandin synthesis. ${ }^{26,27)}$ Recent observations revealed that A.a. infection upregulated the expression of several chemokines, including MCP-1, CCL19, CCL21, and CCR7, which mediate the recruitment of macrophages. ${ }^{28)}$ Our findings suggest that a specific periodontal pathogen, A.a., plays a pivotal role in the development of ventricular remodeling after MI through enhanced macrophage infiltration. However, the numbers of MCP-1 positive cells were statistically comparable between the two groups. Thus, further experiments are needed to elucidate the mechanism.

Elucidation of the mechanisms linking periodontal infection and ischemia-induced myocardial remodeling may help in the development of new clinical strategies for the prevention and treatment of MI patients with periodontitis.

\section{ACKNOWLEDGMENTS}

We thank Ms. Noriko Tamura, Ms. Yasuko Matsuda, and Mr. Kazuya Suzuki for their excellent technical assistance in the experiments.

\section{REFERENCES}

1. Ertl G, Frantz S. Healing after myocardial infarction. Cardiovasc Res 2005; 66: 22-32. (Review)

2. Fischer P, Hilfiker-Kleiner D. Survival pathways in hypertrophy and heart failure: the gp130-STAT3 axis. Basic Res Cardiol 2007; 102: 279-97. (Review)

3. Pfeffer JM, Pfeffer MA, Braunwald E. Influence of chronic captopril therapy on the infarcted left ventricle of the rat. Circ Res 1985; 57: 84-95.

4. Skyschally A, Leineweber K, Gres P, Haude M, Erbel R, Heusch G Coronary microembolization. Basic Res Cardiol 2006; 101: 37382. (Review)

5. Przyklenk K, Connelly CM, McLaughlin RJ, Kloner RA, Apstein CS. Effect of myocyte necrosis on strength, strain, and stiffness of isolated myocardial strips. Am Heart J 1987; 114: 1349-59.

6. Mann DL, Young JB. Basic mechanisms in congestive heart failure. Recognizing the role of proinflammatory cytokines. Chest 1994; 105: 897-904. (Review)

7. De Gennaro L, Brunetti ND, Cuculo A, Pellegrino PL, Di Biase M. Systemic inflammation in nonischemic dilated cardiomyopathy. Heart Vessels 2008; 23: 445-50.

8. Chrysohoou C, Pitsavos C, Barbetseas J, et al. Chronic systemic inflammation accompanies impaired ventricular diastolic function, detected by Doppler imaging, in patients with newly diagnosed systolic heart failure (Hellenic Heart Failure Study). Heart Vessels 2009; 24: 22-6.

9. Frangogiannis NG. The immune system and cardiac repair. Pharmacol Res 2008; 58: 88-111. (Review)

10. Tonetti MS. Periodontitis and risk for atherosclerosis: an update on intervention trials. J Clin Periodontol 2009; 36: 15-9. (Review)

11. Paster BJ, Olsen I, Aas JA, Dewhirst FE. The breadth of bacterial diversity in the human periodontal pocket and other oral sites. Periodontol 2000 2006; 42: 80-7. (Review)

12. Nishihara T, Koseki T. Microbial etiology of periodontitis. Periodontol 2000 2004; 36: 14-26. (Review)

13. Papapanou PN. Periodontal diseases: epidemiology. Ann Periodontol 1996; 1: 1-36. (Review)

14. Henderson B, Nair SP, Ward JM, Wilson M. Molecular pathogenicity of the oral opportunistic pathogen Actinobacillus actinomycetemcomitans. Annu Rev Microbiol 2003; 57: 29-55. (Review)

15. Johnson JD, Chen R, Lenton PA, Zhang G, Hinrichs JE, Rudney JD. Persistence of extracrevicular bacterial reservoirs after treatment of aggressive periodontitis. J Periodontol 2008; 79: 2305-12.

16. Sakurai K, Wang D, Suzuki J, et al. High incidence of actinobacillus actinomycetemcomitans infection in acute coronary syndrome. Int Heart J 2007; 48: 663-75.

17. Genco CA, Arko RJ. Animal chamber models for study of hostparasite interactions. Methods Enzymol 1994; 235: 120-40. (Review)

18. Ogawa M, Suzuki J, Hishikari K, Takayama K, Tanaka H, Isobe M. Clarithromycin attenuates acute and chronic rejection via matrix metalloproteinase suppression in murine cardiac transplantation. J Am Coll Cardiol 2008; 51: 1977-85.

19. Timmers L, Sluijter JP, van Keulen JK, et al. Toll-like receptor 4 mediates maladaptive left ventricular remodeling and impairs cardiac function after myocardial infarction. Circ Res 2008; 102 : $257-64$.

20. Mattila KJ, Nieminen MS, Valtonen VV, et al. Association between dental health and acute myocardial infarction. BMJ 1989; 298: 779-81.

21. Pfeffer MA, Pfeffer JM, Fishbein MC, et al. Myocardial infarct size and ventricular function in rats. Circ Res 1979; 44: 503-12.

22. Morel DW, DiCorleto PE, Chisolm GM. Modulation of endotoxin-induced endothelial cell toxicity by low density lipoprotein. Lab Invest 1986; 55: 419-26.

23. Kerttula Y, Weber TH. Serum lipids in viral and bacterial meningitis. Scand J Infect Dis 1986; 18: 211-5.

24. Rasi V, Ikkala E, Valtonen V. Plasma beta-thromboglobulin in severe infection. Thromb Res 1982; 26: 267-74.

25. Osterud B, Bjørklid E. The production and availability of tissue thromboplastin in cellular populations of whole blood exposed to various concentrations of endotoxin. An assay for detection of endotoxin. Scand J Haematol 1982; 29: 175-84.

26. Semeraro N, Lattanzio A. Interaction of platelets with bacterial endotoxins. Agents Actions 1983; 13: 461-9. (Review)

27. Stuart MJ. Effect of endotoxin on arachidonic acid release and thromboxane B2 production by human platelets. Am J Hematol 1981; 11: 159-64.

28. Zhang T, Kurita-Ochiai T, Hashizume T, Du Y, Oguchi S, Yamamoto M. Aggregatibacter actinomycetemcomitans accelerates atherosclerosis with an increase in atherogenic factors in spontaneously hyperlipidemic mice. FEMS Immunol Med Microbiol 2010; 59: 143-51 